\title{
BMJ Open Pentoxifylline in diabetic kidney disease (VA PTXRx): protocol for a pragmatic randomised controlled trial
}

\author{
David J Leehey (D , ${ }^{1,2}$ Kimberly Carlson, ${ }^{3}$ Domenic J Reda, ${ }^{3}$ Ian Craig, ${ }^{4}$ \\ Christina Clise, ${ }^{5}$ Todd A Conner, ${ }^{5}$ Rajiv Agarwal, ${ }^{6}$ James S Kaufman, ${ }^{7}$ \\ Robert J Anderson, ${ }^{8}$ Douglas Lammie, ${ }^{3}$ Jeffrey Huminik, ${ }^{5}$ Linda Polzin, ${ }^{1}$ \\ Conor McBurney, ${ }^{1}$ Grant D Huang, ${ }^{9}$ Nicholas V Emanuele ${ }^{1}$
}

To cite: Leehey DJ, Carlson K, Reda DJ, et al. Pentoxifylline in diabetic kidney disease (VA PTXRx): protocol for a pragmatic randomised controlled trial. BMJ Open 2021;11:053019. doi:10.1136/ bmjopen-2021-053019

- Prepublication history and additional online supplemental material for this paper are available online. To view these files, please visit the journal online. To view these files, please visit the journal online (http://dx.doi.org/10.1136/ bmjopen-2021-053019).

Received 03 May 2021 Accepted 16 June 2021
Check for updates

(C) Author(s) (or their employer(s)) 2021. Re-use permitted under CC BY-NC. No commercial re-use. See rights and permissions. Published by BMJ.

For numbered affiliations see end of article.

Correspondence to Dr David J Leehey; david.leehey@va.gov

\section{ABSTRACT}

Introduction Diabetic kidney disease (DKD) is the most frequent cause of end-stage renal disease (ESRD) in the USA and worldwide. Recent experimental and clinical data suggest that the non-specific phosphodiesterase inhibitor pentoxifylline (PTX) may decrease progression of chronic kidney disease. However, a large-scale randomised clinical trial is needed to determine whether PTX can reduce ESRD and death in DKD.

Methods and analysis Veterans Affairs (VA) PTXRx is a pragmatic, randomised, placebo-controlled multicentre VA Cooperative Study to test the hypothesis that PTX, when added to usual care, leads to a reduction in the time to ESRD or death in patients with type 2 diabetes with DKD when compared with usual care plus placebo. The study aims to enrol 2510 patients over a 4-year period with an additional up to 5-year follow-up to generate a total of 646 primary events. The primary objective of this study is to compare the time until ESRD or death (all-cause mortality) between participants randomised to PTX or placebo. Secondary endpoints will be: (1) health-related quality of life, (2) time to doubling of serum creatinine, (3) incidence of hospitalisations for congestive heart failure,

(4) incidence of a three-point major adverse cardiovascular events composite (cardiovascular death, non-fatal myocardial infarction, non-fatal stroke), (5) incidence of peripheral vascular disease, (6) change in urinary albuminto-creatinine ratio from baseline to 6 months and (7) rate of annual change in estimated glomerular filtration rate (eGFR) during the study period.

Ethics and dissemination This study was approved by the VA Central Institutional Review Board (cIRB/18-36) and will be conducted in compliance with the Declaration of Helsinki and the Guidelines for Good Clinical Practice.

The Hines Cooperative Studies Programme will finalise the study results, which will be published in accordance with the Consolidated Standards of Reporting Trials statement in a peer-reviewed scientific journal.

Trial registration number NCT03625648.

\section{INTRODUCTION}

Diabetic kidney disease (DKD) is the most frequent cause of chronic kidney disease (CKD) and end-stage renal disease (ESRD) in the USA. ${ }^{1}$ Patients with diabetes with
Strengths and limitations of this study

- Large randomised, controlled, pragmatic, multicentre clinical trial to determine if pentoxifylline (PTX) can prevent end-stage renal disease (ESRD) and/or death in patients with diabetic kidney disease.

- PTX is a readily available, safe and inexpensive medication which might be effectively repurposed to treat this condition.

- Study medication is added to standard of care therapy in both the active intervention and placebo control groups. Standard of care is determined by the treating physicians and not protocolised.

- The primary outcome is time to ESRD or death (allcause mortality), hard endpoints that are clinically significant to patients

- As a Veterans Affairs study, most participants are expected to be male, which could somewhat limit generalisation of the findings to female patients.

ESRD on maintenance dialysis have markedly impaired survival and quality of life (QoL). ${ }^{2}$

The non-specific phosphodiesterase inhibitor pentoxifylline (PTX) was approved by the US Food and Drug Administration (FDA) in 1984 for the treatment of peripheral vascular disease (PVD). PTX has been shown in several animal models of kidney disease to reduce proteinuria and preserve renal function. These effects are associated with a reduction in inflammation, oxidative stress and fibrosis. ${ }^{3}$ There are clinical studies supporting a role for PTX in DKD, but they have enrolled a small number of subjects, were of short duration, and used surrogate endpoints such as reduction in proteinuria and changes in eGFR, not hard endpoints such as ESRD and death. ${ }^{4}$ In a recent clinical trial from Spain, PTX reduced loss of eGFR and urinary albumin excretion (UAE) in diabetic patients with stage 3-4 CKD and UAE $>30 \mathrm{mg} / 24$ hours. ${ }^{5}$ There were no serious safety concerns with PTX, only a 2-fold 
Table 1 Care in patients with diabetes and CKD in VA during 2015

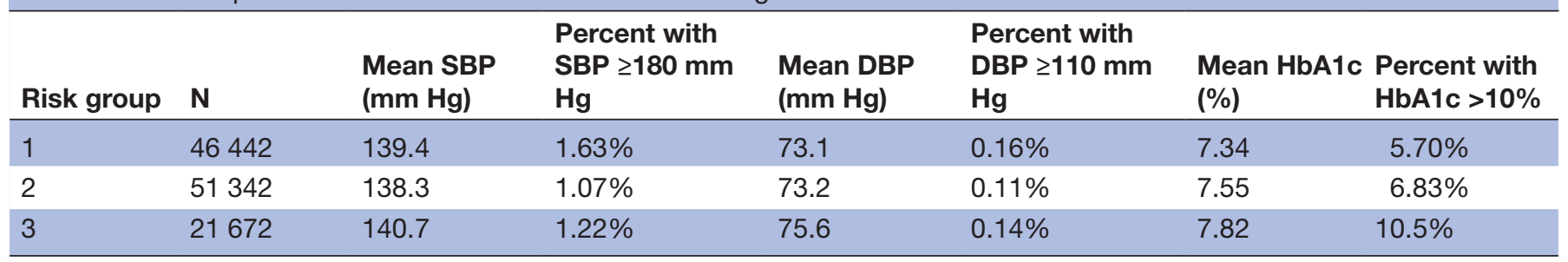

Risk groups:

$1=$ eGFR [15-30) (regardless of proteinuria).

$2=$ eGFR $[30-45)$ with $U A C R \geq 30$.

$3=$ eGFR [45-60) with UACR $\geq 300$.

Data are from calendar year 2015 and include SBP, DBP and HbA1c.

CKD, chronic kidney disease; DBP, diastolic blood pressure; eGFR, estimated glomerular filtration rate; HbA1c, glycosylated haemoglobin;

SBP, systolic blood pressure; UACR, urinary albumin-to-creatinine ratio; VA, veterans affairs.

increased incidence of mild digestive symptoms noted. These findings suggest that PTX could be an effective and safe therapeutic option for treatment of DKD. Nevertheless, more definitive studies, with hard endpoints on efficacy and safety, are needed.

\section{STUDY DESIGN}

Veterans Affairs (VA) PTXRx is a pragmatic, randomised, controlled, parallel, multicentre clinical trial to test the hypothesis that PTX, when added to usual care, leads to a reduction in the incidence of ESRD or death in patients with type 2 diabetes with DKD when compared with usual care plus placebo. The study will recruit Veteran patients from up to $40 \mathrm{VA}$ hospitals throughout the USA with an expected treatment duration of up to 9 years. This study will use a pragmatic design, and other aspects of patient care will not be protocolised. It was believed that it was unnecessary to do so, as the VA has been acknowledged to meet or exceed national standards of care in the care of diabetic patients. ${ }^{6-8}$ In a database analysis of patients with diabetes with CKD, we looked at the three high risk groups for ESRD which will be included in VA PTXRx (see the Methods section). We found that control of blood pressure and blood glucose in these patients adhered to American Diabetes Association (ADA) guidelines (table 1). In addition, the study has been designed with minimal clinic visits and laboratory testing to reduce patient burden and study withdrawals. Other than randomisation to PTX or matched placebo in a 1:1 ratio, patient care will be handled by uual providers according to recommended standards of care. The study has been approved by the VA Central Institutional Review Board and is currently enrolling participants in the ramp-up phase since November of 2019.

\section{OUTCOMES}

\section{Primary outcome}

The primary objective of this study is to compare the time until ESRD or death (all-cause mortality) between participants randomised to PTX or placebo. ESRD will be defined as institution of chronic dialysis or renal transplantation. Chronic dialysis will be defined as any of the following: (1) dialysis for $>90$ days, (2) dialysis for $>30$ days and $\leq 90$ days, if the participant dies between 30 and 90 days, (3) dialysis for $\leq 30$ days if the patient dies in $\leq 30$ days, if it is determined by the adjudication committee that the patient would not have recovered renal function. This determination will be based primarily on the presence or absence of severe (eGFR $<30)$ proteinuric DKD prior to need for dialysis, since such patients are unlikely to recover renal function. ${ }^{9}$

ESRD and death are the two most clinically relevant endpoints in patients with CKD. However, the definition of ESRD has not been consistent among clinical trials. ${ }^{10}$ We opted to use renal replacement therapy as the primary renal outcome. In previous trials of renin-angiotensinaldosterone (RAAS) blockade and sodium glucose cotransporter 2 (SGLT2) inhibition in DKD, composite primary endpoints including hard (ESRD and death) and surrogate (doubling of serum creatinine, changes in eGFR) outcomes have been employed. ${ }^{11}{ }^{12}$ However, surrogate outcomes, as opposed to ESRD or death, do not have the same degree of clinical importance to patients. Nonetheless, we have recognised the necessity of including only high-risk patients for ESRD and using a prolonged (at least 5 years) follow-up period, longer than in previous trials.

\section{Secondary outcomes}

Secondary outcomes that will be compared include (1) health-related QoL (using the RAND Corporation Kidney Disease Quality of Life-Short Form instrument), (2) time to doubling of serum creatinine from baseline (corresponding to a decline in eGFR of $\geq 50 \%$ ), (3) incidence of hospitalisation for congestive heart failure (CHF), (4) incidence of a three-point major adverse cardiovascular event (MACE) composite, including: cardiovascular death, non-fatal myocardial infarction, non-fatal cerebrovascular event (stroke), (5) incidence of a PVD event (limb revascularisation or non-traumatic limb amputation), (6) change in urine albumin to creatinine ratio (UACR) 
from baseline to 6 months and (7) rate of annual change in eGFR during the study period. Safety (serious adverse events (SAEs), adverse events (AEs) possibly or probably related to study drug, or discontinuation of study drug) will also be a secondary outcome.

The QoL assessment was included since this is a patientcentred trial and both severe CKD and ESRD have a marked adverse effect on QoL. ${ }^{13}$ In addition, QoL is underinvestigated and should be used more in trials. ${ }^{14}$ Time to doubling of serum creatinine will be a secondary outcome as it is a surrogate for development of ESRD. Hospitalisation for CHF is justified since CHF is the most common adverse cardiovascular outcome in CKD. A cardiovascular MACE composite is justified because of the markedly increased risk of cardiovascular disease in patients with renal disease and potential cardiovascular benefits if PTX slows worsening of renal function. PVD was included since PTX is FDA-approved for this condition. Change in UACR from baseline is justified since studies have shown that this is a predictor of renal outcomes. ${ }^{15}$ Rate of change in eGFR per year during the study period is a reasonable predictor of the ultimate development of ESRD.

Previous clinical trials in DKD have generally looked at cardiovascular events as secondary outcomes because the risk of cardiovascular events increases as renal function worsens. ${ }^{16} 17$ Therefore, an intervention that improves renal outcomes may also improve cardiovascular outcomes. Clinical trials of RAAS blockade in DKD that have found benefits in terms of renal outcomes did not necessarily show improvement in cardiovascular outcomes, ${ }^{11}$ although SGLT2 inhibitors have been shown to improve both renal and cardiovascular outcomes. ${ }^{12}$ Ultimately, we believed that inclusion of cardiovascular endpoints in the primary outcome was not advisable, considering contrary to RAAS blockers and SGLT2 inhibitors, we could find no evidence of a cardioprotective effects of PTX in the literature.

\section{METHODS}

Inclusion criteria will be the presence of both type 2 diabetes and CKD stage 3 or 4 (eGFR $15-60 \mathrm{~mL} / \mathrm{min} / 1.73$ $\mathrm{m}^{2}$ ). Participants need to be in one of the following categories at the time of randomisation and on one or more occasions 3 months or more prior to randomisation:

- eGFR 15 to less than $30 \mathrm{~mL} / \mathrm{min} / 1.73 \mathrm{~m}^{2}$.

- eGFR 30 to less than $45 \mathrm{~mL} / \mathrm{min} / 1.73 \mathrm{~m}^{2}$ with UACR $>30 \mathrm{mg} / \mathrm{g}$.

- eGFR 45 to less than $60 \mathrm{~mL} / \mathrm{min} / 1.73 \mathrm{~m}^{2}$ with UACR $>300 \mathrm{mg} / \mathrm{g}$.

Exclusion criteria are listed in box 1 .

\section{Why were the inclusion and exclusion criteria chosen?}

The inclusion criteria are based on the Kidney Disease: Improving Global Outcomes categorical meta-analysis (adjusted relative risk), commonly known as the heat map, ${ }^{18}$ which gives relative risks of ESRD and mortality

\section{Box 1 Exclusion criteria}

1. Type 1 diabetes.

2. History of non-diabetic kidney disease.

3. Severe comorbid conditions expected to reduce life expectancy to less than 1 year, as determined by LSI.

4. Active substance abuse, homelessness or other condition that is likely to result in participant non-compliance as determined by the LSI.

5. Previous organ or bone marrow transplant.

6. Pregnancy, breast feeding or female of childbearing potential unwilling to use a reliable form of contraception.

7. A recent (within 3 months) cerebral haemorrhage.

8. Current use of oral pentoxifylline.

9. Hypersensitivity to pentoxifylline or any of the components of the formulation.

10. Current use of ketorolac (contraindicated with pentoxifylline).

11. Current use of riociguat (contraindicated with pentoxifylline).

12. Current use of dialysis.

13. Unable to provide informed consent.

14. Any condition that in the opinion of the LSI would make the potential participant non-compliant.

based on eGFR and UACR in patients with CKD. We are including only patients in the highest risk zones of the heat map for ESRD.

\section{Why are we not requiring that patients take RAAS blockers or SGLT2 inhibitors?}

According to ADA guidelines, angiotensin converting enzyme inhibitors (ACEIs) or ARBs are the preferred firstline agent for blood pressure treatment among patients with diabetes, hypertension, eGFR $<60 \mathrm{~mL} / \mathrm{min} / 1.73$ $\mathrm{m}^{2}$, and UACR $\geq 300 \mathrm{mg} / \mathrm{g}$ Cr because of their proven benefits for prevention of ESRD and major cardiovascular events. In the setting of lower levels of albuminuria (30-299 mg/g Cr), ACEI or ARB therapy reduces cardiovascular events but has not been demonstrated to prevent progression to ESRD. In patients without albuminuria, there are no clinical trials to determine whether ACEIs or ARBs improve renal outcomes. Since our study will include patients in all of the above categories, we cannot expect that they will all be treated with RAAS blockers.

We have analysed data from the VA Corporate Data Warehouse (CDW) on the use of ACEI/ARB in the VA during calendar year 2015, and these data are displayed in table 2. Our findings are in accord with data on utilisation with ACEI and ARB outside the VA. ${ }^{19-22}$ Although we are not mandating the use of RAAS blockade, we will stratify for utilisation and conduct a subgroup analyses examining the primary endpoint in those with and without RAAS blockade at baseline.

SGLT2 inhibitors were not in common use at the time of the design of this trial. Since, prescribing patterns have increased due to expanding criteria for use. However, these medications are generally avoided in patients with severe CKD (eGFR <30), which will make up a large portion of the patients in this trial. 


\begin{tabular}{lll} 
Table 2 & Use of ACEl or ARB in VA during 2015 \\
\hline Risk group & N & \% Using ACEI or ARB \\
\hline 1 & 19386 & 41.74 \\
2 & 27384 & 53.34 \\
3 & 13949 & 64.36 \\
Total & 60719 & 50.83 \\
\hline
\end{tabular}

Risk groups:

$1=$ eGFR 15 to less than 30 (regardless of proteinuria)

$2=$ eGFR 30 to less than 45 with UACR $\geq 30$.

$3=e$ GFR 45 to less than 60 with UACR $\geq 300$.

Data are from calendar year 2015 for any use of ACEI or ARB.

ACEI, ACE inhibitors; ARB, angiotensin receptor blockers; eGFR, estimated glomerular filtration rate; VA, Veterans Affairs.

\section{Randomisation}

Randomisation will be stratified according to study site, use of RAAS blockers, and CKD risk category. The treatment allocation ratio, generated by the Hines VA Cooperative Studies Programme (CSP), for the two treatment groups is 1:1 using permuted block scheme with random block size. Eligible participants will be randomly assigned in a double-blind fashion to PTX or placebo using a studyspecific web-based randomisation system maintained by the CSP Clinical Research Pharmacy Coordinating Centre (CSPCRPCC).

\section{Treatment regimens}

Patients will receive PTX $400 \mathrm{mg}$ extended release (ER) tablets once per day or matching placebo starting at randomisation. At the first follow-up visit, approximately 5 weeks after randomisation, the PTX dose will be increased to PTX $400 \mathrm{mg}$ ER tablets two times per day (a maximum daily dose of $800 \mathrm{mg}$ ) or matching placebo as tolerated. Drug titration is implemented to minimise possible gastrointestinal side effects of the medication. If a participant develops intolerance while taking the study medication at the max dose, $400 \mathrm{mg}$ ER two times per day dose, then the regimen may be reduced to $400 \mathrm{mg}$ ER once daily. If the intolerance dissipates and does not reappear after 1 month, a rechallenge of the max dose, $400 \mathrm{mg}$ ER two times per day, can be initiated. If the intolerance reappears, the participant can be maintained at the reduced dose, $400 \mathrm{mg}$ ER tablets once daily, for the remainder of study participation.

If the intolerance continues at the $400 \mathrm{mg}$ ER once daily dosing, the study medication will be discontinued. However, every effort should be made to minimise permanent discontinuation of study medication or withdrawal of patients from the study. Temporary suspension or permanent discontinuation of study medication may occur under the following circumstances: (1) medication intolerance, (2) pregnancy or breast feeding by participant, (3) decision of investigator or participant to discontinue study treatment for any reason. If the condition leading to a suspension of study medication resolves and deemed reasonable by the investigator and acceptable by the participant, the participant should be restarted on study medication at a previously well-tolerated dose. Whenever study treatment is suspended or discontinued, it will be documented on the Study Drug Prescription Dosage Change form.

In clinical trials to date, daily doses of PTX from 400 to $1200 \mathrm{mg}$ daily have been employed. PTX is metabolised by the liver to both inactive and active metabolites. In CKD patients, there is accumulation of an active metabolite of PTX (Metabolite V), but not the parent drug. In the only study specifically looking at pharmacokinetics in CKD patients, the authors recommended a dosage reduction from $600 \mathrm{mgtwo}$ times per day to $400 \mathrm{mg}$ two times per day for patients with moderate renal impairment and 200-400 mg/day for severe renal impairment. ${ }^{23}$ These doses are consistent with those listed in published dosage guidelines $^{24}$ :

- Creatinine clearance $>50 \mathrm{~mL} / \mathrm{min}$ : $400 \mathrm{mg}$ every 8-12 hours.

- Creatinine clearance 10-50 mL/min: $400 \mathrm{mg}$ every 12-24 hours.

- Creatinine clearance <10 mL min: $400 \mathrm{mg}$ every 24 hours.

We are aware that the PREDIAN (Pentoxifylline for Renoprotection in Diabetic Nephropathy) trial $^{5}$ as well as the study of Han et $a l,{ }^{25}$ the largest trial to date, used a daily dose of $1200 \mathrm{mg}$. However, since we will be studying patients with moderate to severe CKD, we will not exceed the $800 \mathrm{mg}$ daily dose.

PTX has a very favourable safety profile, with gastrointestinal disturbances (eg, dyspepsia, nausea, vomiting) being the most commonly reported adverse effect in studies comparing PTX to placebo. The PTX package insert lists 'recent cerebral or retinal haemorrhage' as a contraindication to its use. While recent (within 90 days) cerebral haemorrhage will be an exclusion criterion, we were concerned that a retinal haemorrhage exclusion would affect the generalisability of the study, as a substantial portion of diabetic patients with $\mathrm{CKD}$ have coexistent retinal disease.

We spent considerable effort gathering information about the association of PTX with retinal haemorrhage. Neither the various manufacturers of PTX nor the FDA were able to provide any data on which this contraindication was based. A search of the FDA's Adverse Event Reporting System database discovered only 11 cases of retinal haemorrhage reported in the past 30 years. There is no literature that indicates an increased risk of retinal haemorrhage with PTX. One paper actually suggested that PTX might reduce neovascularisation and promote absorption of haemorrhage. ${ }^{26}$ FDA has approved our Investigational New Drug (IND) application to omit the exclusion of recent retinal haemorrhage. The study will have a Data Monitoring Committee (DMC), which will be able to determine if there is an increased risk of retinal haemorrhage in patients receiving PTX.

In addition, it is possible that PTX may increase bleeding event risk, especially in high-risk patients. Therefore, we 
will track use of anticoagulant and antiplatelet medications at each study visit and the DMC will closely monitor study patients for bleeding events. Careful attention will also be paid at each clinic visit to other medications which are either contraindicated with PTX (ketorolac, riociguat) or can interact with PTX (PTX can increase plasma levels of theophylline).

\section{Study visits}

The first visit (Baseline) will focus on inclusion and exclusion criteria and medication review (including dosages of all anti-diabetic and antihypertensive medications) in addition to randomisation within up to 5 days if eligibility criteria is met. The first follow-up visit ( 5 weeks \pm 1 week from randomisation) will be focused on adherence and possible adverse effects from study medication and titration to maximal dose as tolerated. Subsequent visits will occur quarterly. They may be conducted either in person or by phone, and will focus on capture of potential endpoints, SAEs (hospitalisations, life-threatening illnesses or a new or worsening clinical condition requiring acute intervention) and study-related AEs. Health-related QoL will be assessed on a yearly basis. An end-of-trial visit will be conducted in person for all participants who have not reached a primary endpoint. All study medications will be shipped directly to the study patients using a well-established VA infrastructure (VA Consolidated Mail Outpatient Pharmacy). Pill counts will not be employed, though participants will be asked to return unused medication. There will be no prohibited care or intervention during the trial other than the contraindicated medication listed in the exclusion criteria. It is expected that patients will be enrolled for 4 years and followed for up to 9 years (table 3 ).

\section{STATISTICAL ANALYSIS}

The key statistical assumption is that occurrence of the primary endpoint occurs in $26.6 \%$ of the placebo group and $21.6 \%$ of the PTX group at 6 years, and a relative risk reduction of $19 \%$ for PTX compared with the placebo group is considered a clinically important effect. The study aims to randomise 2510 participants to either PTX or placebo and will be performed at $40 \mathrm{VA}$ research sites over 4 years of recruitment. It is anticipated that this sample size will generate a total of 646 primary events by the end of the study.

Based on a meta-analysis demonstrating that each $30 \%$ reduction in albuminuria translates to a $23.7 \%$ decrease in the risk of ESRD, ${ }^{27}$ we sought to quantify the expected reduction of ESRD by PTX. In small clinical trials using PTX, the average reduction in albuminuria due to PTX was $32.1 \%$, which translates to a projected $25.4 \%$ decrease in the risk of ESRD. ${ }^{55^{28-31}}$ As the proposed study will be the first to examine the effect of PTX compared with placebo on time to development of ESRD or death, it is powered to detect a $19 \%$ relative reduction in the 6 years event rate for the PTX group, compared with the placebo group. This effect size is commensurate with observed reductions in event rates from other comparable renoprotective studies. For example, the RENAAL trial demonstrated that treatment with losartan reduced the risk of ESRD by $28 \%(\mathrm{p}=0.0002)$, and it reduced the risk of ESRD or death by $20 \%(\mathrm{p}=0.01) .{ }^{11}$

The expected event rates for the control group were estimated using retrospective data from a cohort of Veterans meeting study inclusion criteria obtained from the VA CDW. Using ICD diagnoses codes and laboratory results, a dataset was compiled of all Veterans matching the inclusion criteria between 1 January 2008 and 31 December 2010 with respect to type $2 \mathrm{DM}$ and CKD risk group. A total of 78718 potentially eligible Veterans were allocated to one of the three risk groups ( $\mathrm{N}=38801$ for risk group 1; N=28 838 for risk group 2; $\mathrm{N}=11079$ for risk group 3). This cohort was followed through 2016 to determine how many would have experienced the primary outcome of either death or ESRD. The observed event rates for a primary event based on the amount of follow-up time are shown in table 4 . After 6 years, $26.6 \%$ of Veterans in our retrospective cohort experienced a primary outcome of either death or ESRD.

The primary analysis will compare Kaplan-Meier curves using a two-sided log rank test to test the null hypothesis that the distribution of time to ESRD or death is the same for the two treatment groups. In addition, to support the primary analysis, the proportional hazards assumptions will be assessed and the hazard ratio (HR) will be estimated. Cox regression will be used to assess the treatment effect as well as to adjust for baseline participant characteristics and to examine treatment by characteristic interactions. A preplanned subgroup analysis of the primary outcome will also be conducted to compare time to first occurrence of primary event between treatment groups with each of the randomisation strata. Secondary outcome measures will be organised with a hierarchical structure with a gatekeeping procedure to control the family wise-type I error due to multiple testing. The primary analyses will be on an intention-to-treat basis. All participants will be followed a minimum of 5 years and a maximum of 9 years. Average follow-up will be approximately to 6.7 years per participant. One non-binding interim analysis has been planned a-priori to examine both efficacy and futility and will be conducted when $50 \%$ (323) of primary events have occurred. If either of the thresholds is met for futility or efficacy, this will trigger a DMC discussion on whether to stop or continuing to enrol participants. If neither of the thresholds is met at the interim analysis, the study will continue.

Given the complexities of implementing a large-scale trial, we built in a ramp-up phase starting at six sites during the initial 12 months. The ramp-up phase intends to assess and streamline the recruitment process, determine the most effective recruitment strategies, and identify best processes to be used by site coordinators at future centres. We plan to recruit based on patient lists generated by the VA CDW as well as by local VA Informatics departments in addition to traditional methods. Based on CDW data, there are an adequate number of patients to obtain the desired sample size. 
Table 3 Study procedures

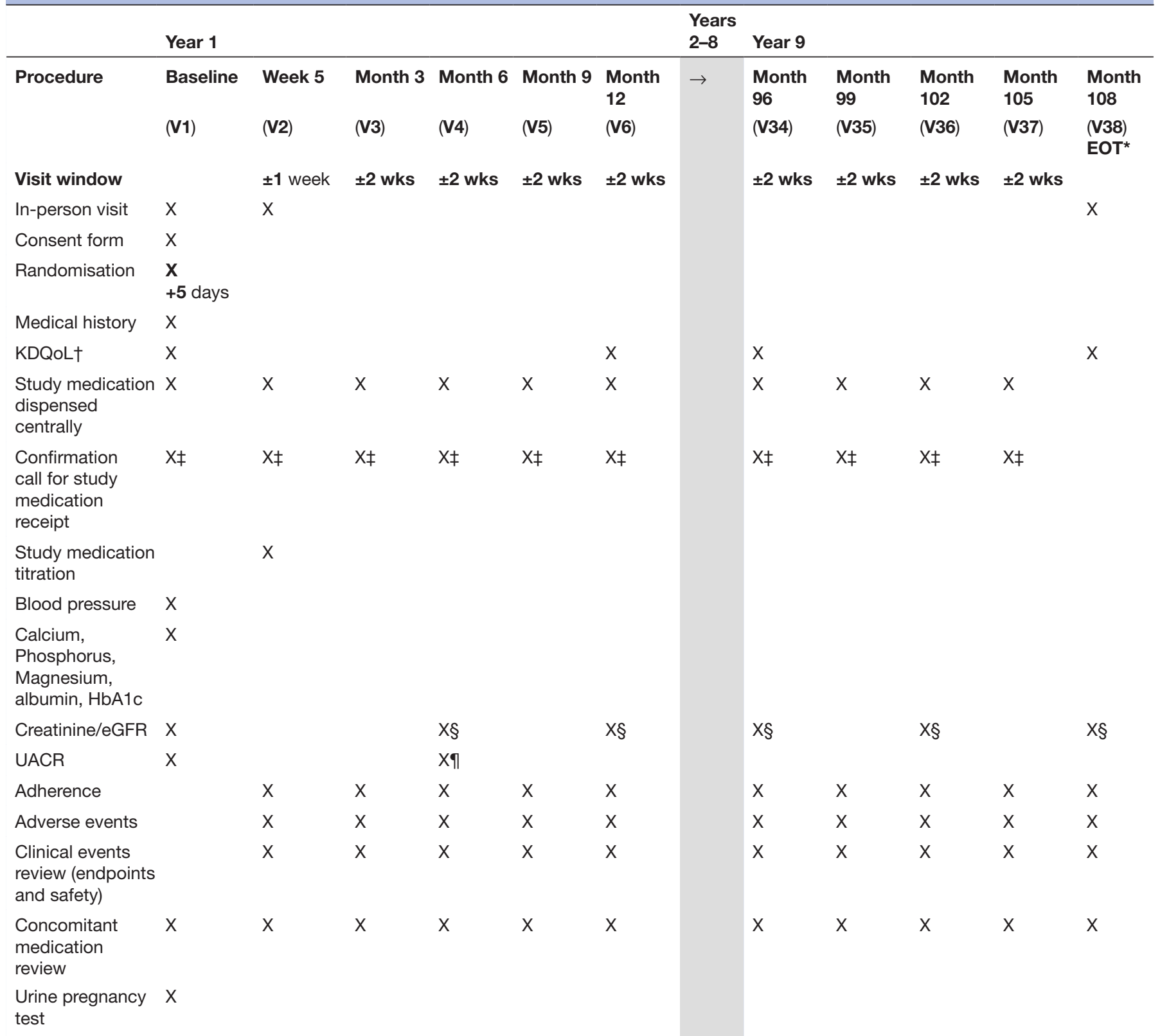

*EOT visit will not be required if a living participant has experienced dialysis or renal transplantation or is on limited participation.

†KDQoL will be measured at baseline, every 12 months thereafter, and at the end of the trial (V1, V6, V10, V14, V18, V22, V26, V30, V34, V38). $\ddagger$ Confirmation call will be done after medication was dispensed to confirm if patient has received study drug.

§Creatinine/eGFR will be collected every 6 months unless available in the chart within past 3 months (V1, V4, V6, V8, V10, V12, V14, V16, V18, V20, V22, V24, V26, V28, V30, V32, V34, V36, V38).

IIf not available in the chart within past 14 days.

eGFR, estimated glomerular filtration rate; EOT, End of Trial; HbA1c, glycosylated haemoglobin; KDQoL, Kidney Disease Quality of Life; UACR,

urinary albumin-to-creatinine ratio.

\section{QUALITY BY DESIGN CONSIDERATIONS}

VA PTXRx has embedded quality by design principles into the design, start-up and conduct of the trial. This approach that has been widely adopted by pharmaceutical sponsored trials. The goal is to improve the quality and efficiency of the trial by prospectively identifying critical to quality trial factors and developing a plan to periodically monitor these factors. During planning, a risk-based monitoring approach was used to define performance metrics for the trial. These efforts were taken to further enhance the ability to have the trial provide evidence to put findings into practice. ${ }^{32} 33$

\section{ETHICAL CONSIDERATIONS}

This study has been approved by the VA Central Institutional Review Board (cIRB/18-36) and will be conducted under FDA IND oversight and in compliance with the 
Table 4 Event rates by amount of follow-up time

\begin{tabular}{lccc}
\hline $\begin{array}{l}\text { Amount of follow-up } \\
\text { time }\end{array}$ & $\begin{array}{l}\% \text { of participants experiencing } \\
\text { primary endpoint }\end{array}$ & $\begin{array}{l}\text { \% of participants } \\
\text { experiencing ESRD event }\end{array}$ & $\begin{array}{l}\text { \% of participants } \\
\text { experiencing death }\end{array}$ \\
\hline 2 years & 9.4 & 7.4 & 2.0 \\
\hline years & 14.4 & 11.0 & 3.4 \\
\hline years & 18.9 & 13.9 & 5.0 \\
\hline 5 years & 22.8 & 16.1 & 6.7 \\
\hline 6 years & 26.6 & 18.1 & 8.5 \\
\hline 7 years & 29.7 & 19.9 & 9.9 \\
\hline
\end{tabular}

ESRD, end-stage renal disease.

ethical principles of the Declaration of Helsinki, the protocol, and the Guidelines for Good Clinical Practice. Informed consent will be obtained from every participant by study personnel. Surrogate consent will not be allowed. Personal information about potential and enrolled participants will be collected, shared and maintained in a confidential fashion. Protocol modifications will be disseminated to all study stakeholders as a new protocol version as necessary.

\section{Post-trial care and compensation for harm}

If a participant is injured because of taking part in this study, the VA will provide necessary medical treatment at no cost unless the injury was due to non-compliance with study procedures. Participants will continue their care in the VA post-trial.

\section{PATIENT AND PUBLIC INVOLVEMENT}

Veterans were engaged as stakeholders through the CSP Coordinating Centre's (CSPCG) Human Rights Committee (HRC). The HRC meets on an annual basis to review study protocols, both during the design and active phases of the study, to assess progress and provide recommendations regarding human rights issues for Veteran participants. The HRC will also conduct a selected number of site visits to interview study staff and participants regarding the process and personal experiences in recruitment and continued participation to ensure that participants' rights and safety are being properly protected.

\section{ROLES AND RESPONSIBILITIES: COMMITTEES}

The study is managed by the CSPCC located in Hines, IL and the CSPCRPCG in the Albuquerque, NM. There is an Executive Committee (EC), the Cooperative Studies Scientific Evaluation Committee (CSSEC), a HRC and a DMC (see 'Study monitoring and data access' section). The data management team is composed of members of the CSPCC. Blinded, physician members of the EC will adjudicate whether a participant who dies while on dialysis for $<30$ days should be counted as having had a death endpoint or an ESRD endpoint.

\section{DATA COLLECTION PLAN AND DATA MANAGEMENT}

The Hines CSPCC will be responsible for the management and quality control of the study data. Data collection and entry is the responsibility of the study coordinator at each site, under the supervision of the local site investigator. Case report forms and operations manual were developed jointly by the Chairs' Office, the CSPCRPCC and the Hines CSPCC. Data will be entered the into an electronic data capture system (DF/Net Research, V.2016.1).

Data management and quality staff at the Hines CSPCC and the CSPCRPCC will further review submitted data for completeness and consistency adding data queries to items that fail these checks. The Hines CSPCG will prepare summary reports for the Study Chair, the DMC and other monitoring groups of the data to track study progress and conduct final analyses of the study data.

\section{Missing data}

We will use VA data sources and federally maintained databases to make primary endpoint determinations for most participants, in particular those lost to active follow-up. In the event of missing data due to withdrawal, participants will be censored at the date of withdrawal.

\section{Data security}

The analytical database will not contain information that can directly identify the study participant. Only CSPapproved individuals will have access to the personal health information of study participants. Neither the Study Chair nor Chair's office will have access to personal health information.

\section{STUDY MONITORING AND DATA ACCESS}

Data quality and completeness of data retrieval will be closely monitored on an ongoing basis by the CSPCC. The study biostatistician will present interim monitoring reports (overall and by site) to the EC and DMC that will include recruitment of participants, characteristics of the population, completeness of data retrieval, and data quality. All reportable study intervention-related AEs and SAEs (intervention related or not related) will be provided to the DMC on a schedule set by the DMC, but no less than annually. The DMC will determine the need 
for unblinding to treatment assignment based on $\mathrm{AE} / \mathrm{SAE}$ data. The DMC will advise the CSP Director whether the study should continue or be stopped for safety reasons. The trial will also be audited by the VA Site Monitoring, Auditing, and Resource Team (SMART). Monitoring will be a collaboration of onsite site visits conducted by SMART Clinical Research Monitors; remote monitoring performed by SMART and Coordinating Centre Quality Assurance RNs; and centralised statistical monitoring on at least an annual basis, a process independent from the investigators and sponsor. The CSPCC will maintain access to all data throughout the trial. Digital data underlying primary scientific publications from this study will be held as part of a data sharing resource maintained by the CSP. The data may be available through execution of a data use agreement and only under certain conditions consistent with the informed consent and CSP policy, which prioritise protecting subjects' privacy and confidentiality to the fullest extent possible.

\section{ETHICS AND DISSEMINATION}

This study was approved by the VA Central Institutional Review Board (cIRB/18-36) (ID: 1382143) and will be conducted in compliance with the Declaration of Helsinki and the Guidelines for Good Clinical Practice. The Hines CSP will finalise the study results, which will be published in accordance with the Consolidated Standards of Reporting Trials statement in a peer-reviewed scientific journal.

The EC has the authority to establish one or more publication committees, usually made up of selected site investigators and members of the EC, for the purpose of producing manuscripts for presentation and publication. Any presentation or publication needs to be approved by the EC. All publications will list all participating personnel in the study (not necessarily as authors of the manuscript). The full study protocol and informed consent will be made publicly available.

In this manuscript, we used the Standard Protocol Items: Recommendations for Interventional Trials reporting guidelines. ${ }^{34}$

\author{
Author affiliations \\ ${ }^{1}$ Edward Hines Junior VA Hospital, Hines, Illinois, USA \\ ${ }^{2}$ Loyola University Medical Center, Maywood, Illinois, USA \\ ${ }^{3}$ Cooperative Studies Program, Edward Hines Junior VA Hospital, Hines, Illinois, USA \\ ${ }^{4}$ Division of Strategic Innovation, Evaluation, and Communication, Center for Clinical \\ Standards and Quality, Baltimore, Maryland, USA \\ ${ }^{5}$ VA Cooperative Studies Program, Clinical Research Pharmacy Coordinating Center, \\ Albuquerque, New Mexico, USA \\ ${ }^{6}$ Veterans' Affairs Medical Center, Indianapolis, Indiana, USA \\ ${ }^{7}$ Research Service, New York Harbor Health Care System, New York, New York, USA \\ ${ }^{8}$ Veterans' Affairs Medical Center, Omaha, Nebraska, USA \\ ${ }^{9}$ Veterans Affairs Office of Research and Development, Washington, District of \\ Columbia, USA
}

Acknowledgements Preliminary data for this study were obtained with the assistance of VA Informatics and Computing Infrastructure (VINCI), VA HSR HIR 08-204, U.S. Department of Veterans Affairs. (2008). https://vaww.VINCI.med.va. gov.
Contributors DL, KC, DJR, TAC, CC, RA, JSK, RJA, GDH, and NVE designed the study and prepared the manuscript. IC analysed data preparatory to research. DL, $\mathrm{JH}, \mathrm{LP}$ and CM provided managerial assistance. All authors read and approved the final manuscript.

Funding This study is funded by the U.S. Veterans Affairs Cooperative Studies Program [grant number NA].

Competing interests RA has the following general disclosures: Member data safety monitoring committees: Astra Zeneca, Ironwood Pharmaceuticals, Chinook; Member steering committees of randomised trials: Akebia, Bayer, Relypsa, Sanofi and Genzyme US Companies; Member adjudication committees: Bayer, Boehringer Ingelheim; Member scientific advisory board or consultant: Bayer, Relypsa, Reata, Boehringer, Merck, Lexicon, Diamedica.

Patient consent for publication Not required.

Provenance and peer review Not commissioned; externally peer reviewed.

Supplemental material This content has been supplied by the author(s). It has not been vetted by BMJ Publishing Group Limited (BMJ) and may not have been peer-reviewed. Any opinions or recommendations discussed are solely those of the author(s) and are not endorsed by BMJ. BMJ disclaims all liability and responsibility arising from any reliance placed on the content. Where the content includes any translated material, BMJ does not warrant the accuracy and reliability of the translations (including but not limited to local regulations, clinical guidelines, terminology, drug names and drug dosages), and is not responsible for any error and/or omissions arising from translation and adaptation or otherwise.

Open access This is an open access article distributed in accordance with the Creative Commons Attribution Non Commercial (CC BY-NC 4.0) license, which permits others to distribute, remix, adapt, build upon this work non-commercially, and license their derivative works on different terms, provided the original work is properly cited, appropriate credit is given, any changes made indicated, and the use is non-commercial. See: http://creativecommons.org/licenses/by-nc/4.0/.

ORCID iD

David J Leehey http://orcid.org/0000-0002-2078-9948

\section{REFERENCES}

1 Saran R, Li Y, Robinson B, et al. US renal data system 2015 annual data report: epidemiology of kidney disease in the United States. Am J Kidney Dis 2016;67:A7-8.

2 Johansen KL, Dalrymple LS, Glidden D, et al. Association of performance-based and self-reported function-based definitions of frailty with mortality among patients receiving hemodialysis. Clin $\mathrm{J}$ Am Soc Nephrol 2016;11:626-32.

3 Bhanot S, Leehey DJ. Pentoxifylline for diabetic nephropathy: an important opportunity to Re-purpose an old drug? Curr Hypertens Rep 2016;18:8.

4 Shan D, Wu HM, Yuan QY, et al. Pentoxifylline for diabetic kidney disease. Cochrane Database Syst Rev 2012:CD006800.

5 Navarro-González JF, Mora-Fernández C, Muros de Fuentes M, et al. Effect of pentoxifylline on renal function and urinary albumin excretion in patients with diabetic kidney disease: the PREDIAN trial. J Am Soc Nephrol 2015;26:220-9.

6 Asch SM, McGlynn EA, Hogan MM, et al. Comparison of quality of care for patients in the Veterans health administration and patients in a national sample. Ann Intern Med 2004;141:938-45.

7 Kerr EA, Gerzoff RB, Krein SL, et al. Diabetes care quality in the Veterans Affairs health care system and commercial managed care: the triad study. Ann Intern Med 2004;141:272-81.

8 O'Hanlon C, Huang C, Sloss E, et al. Comparing Va and NonVA quality of care: a systematic review. J Gen Intern Med 2017:32:105-21.

9 Lee BJ, Go AS, Parikh R, et al. Pre-admission proteinuria impacts risk of non-recovery after dialysis-requiring acute kidney injury. Kidney Int 2018;93:968-76.

10 Agarwal R. Defining end-stage renal disease in clinical trials: a framework for adjudication. Nephrol Dial Transplant 2016;31:864-7.

11 Brenner BM, Cooper ME, de Zeeuw D, et al. Effects of losartan on renal and cardiovascular outcomes in patients with type 2 diabetes and nephropathy. N Engl J Med 2001;345:861-9.

12 Perkovic V, Jardine MJ, Neal B, et al. Canagliflozin and renal outcomes in type 2 diabetes and nephropathy. N Engl $\mathrm{J} \mathrm{Med}$ 2019;380:2295-306.

13 Mujais SK, Story K, Brouillette J, et al. Health-Related quality of life in CKD patients: correlates and evolution over time. Clin J Am Soc Nephrol 2009;4:1293-301. 
14 Chong K, Unruh M. Why does quality of life remain an underinvestigated issue in chronic kidney disease and why is it rarely set as an outcome measure in trials in this population? Nephrol Dial Transplant 2017;32:ii47-52.

15 Carrero JJ, Grams ME, Sang Y, et al. Albuminuria changes are associated with subsequent risk of end-stage renal disease and mortality. Kidney Int 2017;91:244-51.

16 Bruno G, Merletti F, Bargero G, et al. Estimated glomerular filtration rate, albuminuria and mortality in type 2 diabetes: the Casale Monferrato study. Diabetologia 2007;50:941-8.

17 Amin AP, Whaley-Connell AT, Li S, et al. The synergistic relationship between estimated GFR and microalbuminuria in predicting longterm progression to ESRD or death in patients with diabetes: results from the kidney early evaluation program (keep). Am J Kidney Dis 2013;61:S12-23.

18 Levey AS, de Jong PE, Coresh J, et al. The definition, classification, and prognosis of chronic kidney disease: a KDIGO controversies conference report. Kidney Int 2011;80:17-28.

19 Winkelmayer WC, Fischer MA, Schneeweiss S, et al. Underuse of ACE inhibitors and angiotensin II receptor blockers in elderly patients with diabetes. Am J Kidney Dis 2005;46:1080-7.

20 Cooke CE, Fatodu H. Physician conformity and patient adherence to ACE inhibitors and Arbs in patients with diabetes, with and without renal disease and hypertension, in a Medicaid managed care organization. J Manag Care Pharm 2006;12:649-55.

21 Yang Y, Thumula V, Pace PF, et al. High-Risk diabetic patients in Medicare Part D programs: are they getting the recommended ACEI/ ARB therapy? J Gen Intern Med 2010;25:298-304.

22 Ajiboye O, Segal JB. National trends in the treatment of diabetic nephropathy in the United States. J Clin Pharm Ther 2017;42:311-7.

23 Paap CM, Simpson KS, Horton MW, et al. Multiple-dose pharmacokinetics of pentoxifylline and its metabolites during renal insufficiency. Ann Pharmacother 1996;30:724-9.

24 Brier ME, Aronoff GR, eds. Drug prescribing in renal failure: dosing guidelines for adults and children. 5th ed. Philadelphia, PA: American College of Physicians, 2007.
25 Han SJ, Kim HJ, Kim DJ, et al. Effects of pentoxifylline on proteinuria and glucose control in patients with type 2 diabetes: a prospective randomized double-blind multicenter study. Diabetol Metab Syndr 2015;7:64.

26 Iwafune $\mathrm{Y}$, Yoshimoto $\mathrm{H}$. Clinical use of pentoxifylline in haemorrhagic disorders of the retina. Pharmatherapeutica 1980;2:429-38.

27 Heerspink HJL, Kröpelin TF, Hoekman J, et al. Drug-induced reduction in albuminuria is associated with subsequent renoprotection: a meta-analysis. J Am Soc Nephrol 2015;26:2055-64.

28 Ghorbani A, Omidvar B, Beladi-Mousavi SS, et al. The effect of pentoxifylline on reduction of proteinuria among patients with type 2 diabetes under blockade of angiotensin system: a double blind and randomized clinical trial. Nefrologia 2012;32:790-6.

29 Rodríguez-Morán M, Guerrero-Romero F. Pentoxifylline is as effective as captopril in the reduction of microalbuminuria in non-hypertensive type 2 diabetic patients--a randomized, equivalent trial. Clin Nephrol 2005;64:91-7.

30 Han SJ, Kim HJ, Kim DJ, et al. Effects of pentoxifylline on proteinuria and glucose control in patients with type 2 diabetes: a prospective randomized double-blind multicenter study. Diabetol Metab Syndr 2015;7:64.

31 Navarro JF, Milena FJ, Mora C, et al. Renal pro-inflammatory cytokine gene expression in diabetic nephropathy: effect of angiotensinconverting enzyme inhibition and pentoxifylline administration. Am J Nephrol 2006;26:562-70.

32 Dmitrienko A, Tamhane AC, Bretz F. Multiple testing problems in pharmaceutical statistics. CRC Press, 2009: 30-191.

33 Meeker-O'Connell A, Glessner C, Behm M, et al. Enhancing clinical evidence by proactively building quality into clinical trials. Clin Trials 2016;13:439-44.

34 Chan A-W, Tetzlaff JM, Gøtzsche PC, et al. Spirit 2013 explanation and elaboration: guidance for protocols of clinical trials. BMJ 2013;346:e7586. 